\title{
Observation of a Linearly Dispersing Collective Mode in a Quantum Hall Ferromagnet
}

\author{
I. B. Spielman, ${ }^{1}$ J.P. Eisenstein, ${ }^{1}$ L. N. Pfeiffer, ${ }^{2}$ and K. W. West ${ }^{2}$ \\ ${ }^{1}$ California Institute of Technology, Pasadena, California 91125 \\ ${ }_{2}^{2}$ Bell Laboratories, Lucent Technologies, Murray Hill, New Jersey 07974
}

(Received 21 December 2000; published 2 July 2001)

\begin{abstract}
Double-layer two-dimensional electron systems can exhibit a fascinating collective phase believed to display both quantum ferromagnetism and excitonic superfluidity. This unusual phase has recently been found to exhibit tunneling phenomena reminiscent of the Josephson effect. A key element of the theoretical understanding of this bizarre quantum fluid is the existence of linearly dispersing Goldstone collective modes. Using the method of tunneling spectroscopy, we have demonstrated the existence of these modes. We find the measured velocity to be in reasonable agreement with theoretical estimates.
\end{abstract}

DOI: $10.1103 /$ PhysRevLett.87.036803

The remarkable properties of superfluids and superconductors are intimately related to the existence of a bosonic condensate of composite particles consisting of an even number of fermions within the strongly interacting manybody environment. In superfluid ${ }^{4} \mathrm{He}$ these composite particles are the helium atoms themselves. In superconductors Cooper pairs play the analogous role. In semiconductors excitons, which consist of conduction band electrons paired with valence band holes, have long been considered candidate building blocks of a new class of neutral superfluids [1]. More recently, it has become apparent that double layer two-dimensional electron systems provide yet another system for realizing the exotica of superfluidity. In the presence of a large magnetic field $B$ such systems will exhibit a quantized Hall plateau at $\rho_{x y}=h / e^{2}$ if the layer separation is sufficiently small [2]. When tunneling between the layers is weak this quantum Hall state reflects the condensation of a remarkable strongly interacting electron quantum fluid. This fluid may be viewed as an excitonic superfluid in which an electron in one layer is paired with a hole (in the conduction band) in the other layer. Quantum mechanical uncertainty makes it impossible to tell which layer either component of this composite boson is in. Equivalently, the system may be regarded as a ferromagnet in which every electron exists in a coherent superposition of the "pseudospin" eigenstates which encode the layer degree of freedom [3]. The phase variable of this superposition, which is analogous to the phase variable in conventional superconductors or superfluid ${ }^{4} \mathrm{He}$, determines the orientation of the pseudospin magnetic moment. Spatial variations of the phase govern the low energy excitations in the system. We report here strong evidence, obtained via tunneling spectroscopy, for these excitations.

The physical properties of this bilayer quantum fluid are predicted to be quite exotic [2-6]. For example, the transition to the ordered state is expected to be a finite temperature Kosterlitz-Thouless (KT) transition. This is unusual since the quantum Hall effect (QHE) is usually associated only with a zero temperature quantum phase transition. Below the KT transition temperature, $T_{\mathrm{KT}}$, counterflow superfluidity is expected: transport involving equal but oppo-
PACS numbers: 73.43.Jn, 71.10.Pm

sitely directed currents in the two layers should be dissipationless, at least in linear response [2,4]. There have also been controversial predictions of a Josephson effect in the tunneling transport between the layers $[5,6]$. This issue, in particular, has come under renewed scrutiny since the experimental discovery of a huge resonant enhancement of the zero bias tunneling conductance when the separation between the layers is reduced below a critical value [7].

The Hall plateau $\rho_{x y}=h / e^{2}$ corresponds to total Landau level filling factor $\nu_{T}=1$, with $\nu_{T}$ defined as the ratio of the total electron density $N_{T}$ to the degeneracy $e B / h$ of a single spin resolved Landau level. If the layers are identical, this occurs despite the fact that each layer viewed independently has filling factor $\nu=1 / 2$. That the net system exhibits a QHE even though the layers by themselves do not is a result of the interlayer couplings in the system. If tunneling is negligible the $\nu_{T}=1 \mathrm{QHE}$ results from the interplay of Coulomb interactions among electrons in the same layer with those in opposite layers. If the separation between the layers is too large, the interlayer correlations break down and the QHE disappears.

A good approximation to the ground state at small layer separation is the product of a Slater determinant of all orbital states in the lowest Landau level and a totally symmetric pseudospin wave function [8]. An electron in one layer is pseudospin up, $|\uparrow\rangle$, while one in the other layer is pseudospin down, $|\downarrow\rangle$. Exchange interactions favor each electron being in the same pseudospin state: $|\uparrow\rangle+e^{i \phi}|\downarrow\rangle$. The phase $\phi$ is uniform and, in the absence of tunneling, arbitrary. The system is thus an easy-plane ferromagnet whose moment lies near the $x y$ plane of pseudospin space. Owing to the finite layer separation the pseudospin moment fluctuates, both within the plane and perpendicular to it. The latter corresponds to local fluctuations in the density difference between the layers and are attended by a capacitive energy penalty. These fluctuations become increasingly severe at larger layer spacing and eventually destroy the ordered state. The precise nature of the associated quantum phase transition is still being vigorously investigated [9-11]. Although the ferromagnetic phase has a gap to charged excitations (and hence displays a QHE), it also 
possesses linearly dispersing neutral collective modes (i.e., pseudospin waves) associated with spatial gradients in the phase. These are the Goldstone modes of the broken symmetry ground state and are gapless in the long wavelength limit [5,12]. These modes are the main focus of this Letter.

Figure 1 shows the measured tunneling conductance $d I / d V$ and current $I$ vs the voltage difference $V$ between two $2 \mathrm{D}$ electron gas layers at $\nu_{T}=1$ at $T=25 \mathrm{mK}$. The sample consists of two individually contacted modulationdoped $18 \mathrm{~nm}$ GaAs quantum wells separated by a $9.9 \mathrm{~nm} \mathrm{Al}{ }_{0.9} \mathrm{Ga}_{0.1}$ As barrier layer. Electrostatic gating is used to adjust the layer densities; for the data shown $N_{1}=N_{2}=N_{T} / 2=2.6 \times 10^{10} \mathrm{~cm}^{-2}$. At this density the ratio of interlayer separation $d$ to the magnetic length $\ell=(\hbar / e B)^{1 / 2}$ is $d / \ell=1.61$. This ratio determines the relative importance of interlayer and intralayer Coulomb interactions in the system. As Spielman et al. [7] showed, the sharp peak in $d I / d V$ at $V=0$ disappears when the density, and thus $d / \ell$, is increased. Above $d / \ell \approx 1.84$ the peak in $d I / d V$ is replaced by the familiar deep minimum characteristic of the Coulomb barrier to tunneling between uncorrelated 2D systems [13].

The sharp zero bias peak in $d I / d V$ illustrated in Fig. 1 is accompanied by a near discontinuity in the tunnel current

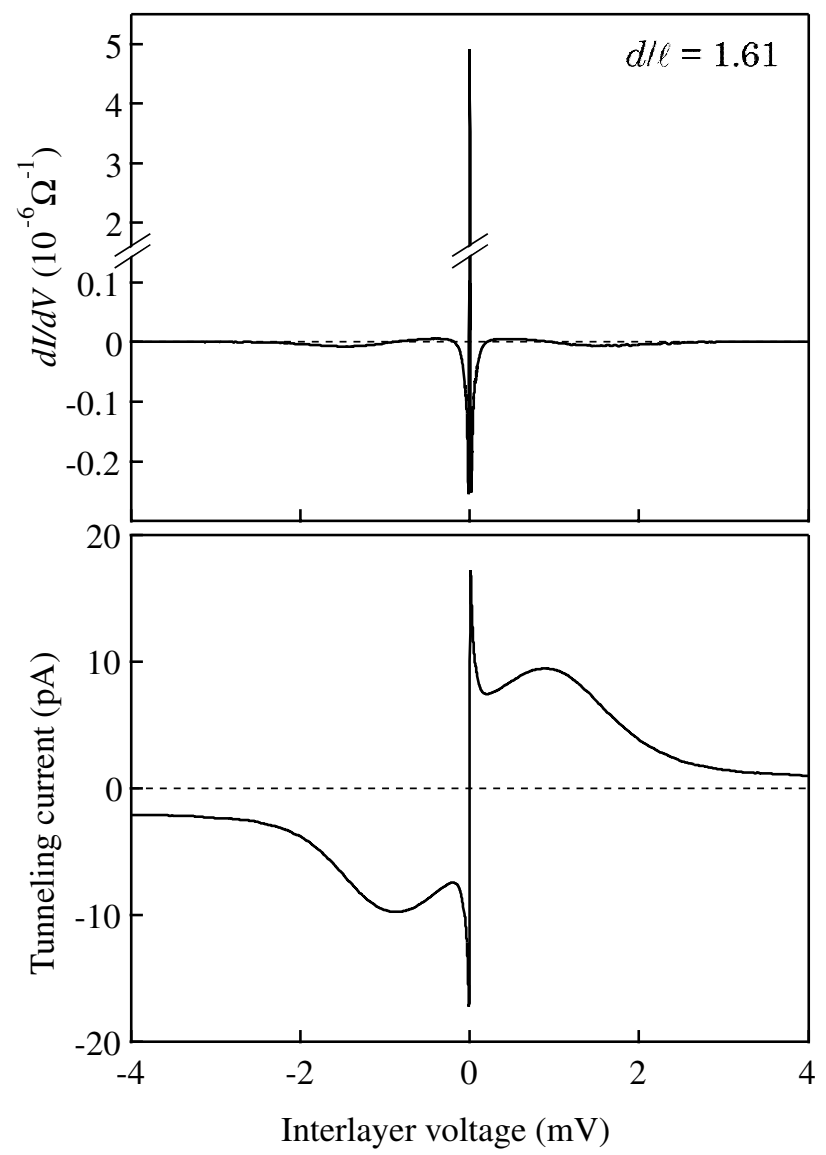

FIG. 1. Tunneling data at $\nu_{T}=1$ and $T=25 \mathrm{mK}$. Total density $N_{T}=5.2 \times 10^{10} \mathrm{~cm}^{-2}$. Upper panel: conductance $d I / d V$ vs interlayer voltage $V$; lower panel: tunnel current $I$ vs $V$.
I. Although this observation is suggestive of a Josephson effect, the present data do not exhibit a true supercurrent at zero bias. The zero bias conductance $G_{0}$, while vastly enhanced over its value at higher densities, remains finite as $T \rightarrow 0$. Similarly, the width $\Gamma$ of the zero bias conductance peak attains a minimum, but nonzero, value in this limit.

Figure 2 illustrates the temperature dependence of the zero bias conductance $G_{0}$ and peak width $\Gamma$ at $d / \ell=1.61$. The peak height rises steadily as the temperature is reduced to around $50 \mathrm{mK}$. Below this temperature it saturates at $G_{0} \approx 5 \times 10^{-6} \Omega^{-1}$. The width $\Gamma$ (defined as the full width at half maximum of the $d I / d V$ peak) decreases down to about the same temperature below which it saturates at $\Gamma \approx 6 \mu \mathrm{V}$. We emphasize that this resonance is roughly 15 times narrower and 150 times taller than the tunnel resonance observed at zero magnetic field in the same sample. At zero field tunneling can be understood in single particle terms and the linewidth reflects the lifetime of the quasiparticles in the 2D systems [14]. In contrast, the dramatic resonance at $\nu_{T}=1$ suggests that a collective mode dominates the spectral weight at low energy.

The low temperature saturation of $G_{0}$ and $\Gamma$ is not understood. Indeed, it is not at all clear that the saturation is intrinsic. Extrinsic electromagnetic interference led to the broader and weaker zero bias peaks reported in the original work of Spielman et al. [7]. Although much effort has since been expended in improving the noise environment, the possibility of a still narrower conductance peak at very low temperature remains. Electron heating is another potential source of the saturation. Finally, we remark that as the present tunneling measurements are effectively two terminal, finite series resistances will ultimately limit height of the tunnel peak.

The Goldstone mode of the coherent QHE ground state offers a natural way to understand the zero bias peak at $\nu_{T}=1$. This mode involves oscillations of the pseudospin magnetization in the $x y$ plane and along the $z$ axis of

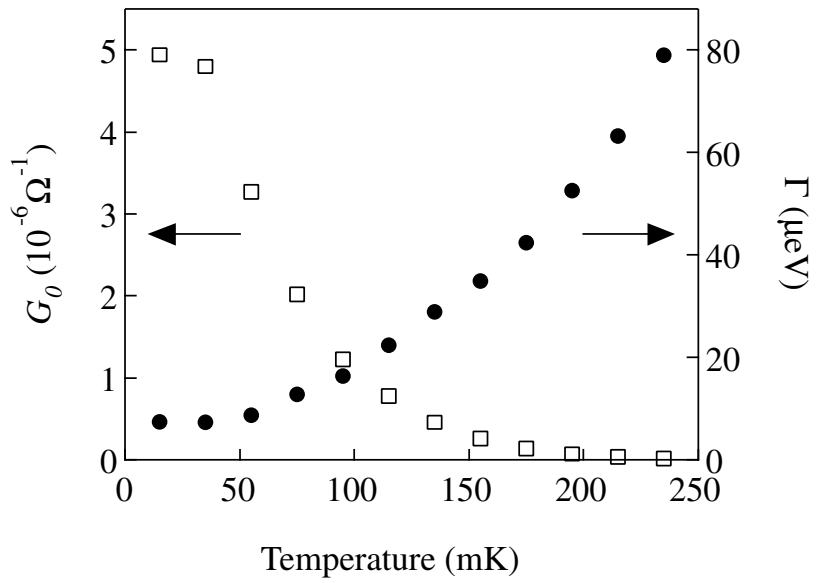

FIG. 2. Linewidth $\Gamma$ (solid dots) and peak height $G_{0}$ (open squares) at $\nu_{T}=1$. Total density $N_{T}=5.2 \times 10^{10} \mathrm{~cm}^{-2}$. 
pseudospin space. In the absence of tunneling, the mode has zero energy at zero wave vector $q$. Any tunneling, however, opens a small gap $\Delta_{0}$ at $q=0$ and allows the mode to effectively transfer charge between the layers. If $\Delta_{0}$ is small enough, a zero bias enhancement of the tunneling conductance is expected. Within this framework, the sharp zero bias peak in $d I / d V$ represents a direct spectroscopic detection of the Goldstone mode of the $\nu_{T}=1$ broken symmetry ground state. The significance of the height and width of this peak is less clear $[15,16]$.

To test this interpretation, we have examined the tunneling spectra after adding a small magnetic field component $B_{\|}$parallel to the 2D planes to the existing perpendicular field $B_{\perp}$. The in-plane field renders the $I-V$ characteristic sensitive to spectral features at the nonzero, and adjustable, wave vector $q=e B_{\|} d / \hbar$. This powerful technique has been applied widely in the past [17] and is of known efficacy in tunneling between $2 \mathrm{D}$ electron systems in semiconductor heterostructures $[18,19]$. In the present circumstance the parallel field has been predicted $[15,16]$ to split the zero bias tunneling peak into two resonances symmetric about $V=0$. The voltage location of these resonances should be $e V= \pm \hbar \omega(q)$, where $\hbar \omega(q)$ is the Goldstone mode energy at the parallel field-induced wave vector $q$. Since the mode disperses linearly for small $q$, detection of the splitting will provide a measure of its velocity $c$.

Figure 3 shows a sequence of tunnel spectra at $\nu_{T}=1$ and $T=25 \mathrm{mK}$ with different parallel fields applied. These data are obtained by tilting the sample relative to an external magnetic field whose magnitude is adjusted to maintain $B_{\perp}$, and hence the Landau level filling factor $\nu_{T}=h N_{T} / e B_{\perp}$ and magnetic length $\ell=$ $\left(\hbar / e B_{\perp}\right)^{1 / 2}$, constant. The parallel field is accurately determined using a second 2D electron gas sample mounted perpendicular to the tunneling sample. The Hall resistance of this second sample is then proportional to $B_{\|}$. The data shown in Fig. 3 again correspond to $N_{1}=N_{2}=N_{T} / 2=5.2 \times 10^{10} \mathrm{~cm}^{-2}$ and $d / \ell=1.61$; similar data have been obtained at various other densities provided $d / \ell<1.84$.

It is clear from Fig. 3 that the parallel magnetic field has a dramatic effect on the tunnel spectrum. Only a few tenths of a tesla are required to strongly suppress the zero bias conductance peak. Closer inspection, however, reveals a more subtle effect: complex structure appears on the flanks of the zero bias peak. This structure, which is magnified in the inset in Fig. 3, first appears as two small peaks in $d I / d V$ positioned symmetrically about $V=0$, superimposed on the still substantial flanks of the main zero bias resonance. As the parallel field increases these split-off peaks move toward higher energies and become more prominent. At the same time the zero bias resonance weakens steadily. As Fig. 3 shows, the split-off peaks are really part of a more complex undulation in the conductance. Qualitatively, these split-off resonances have the

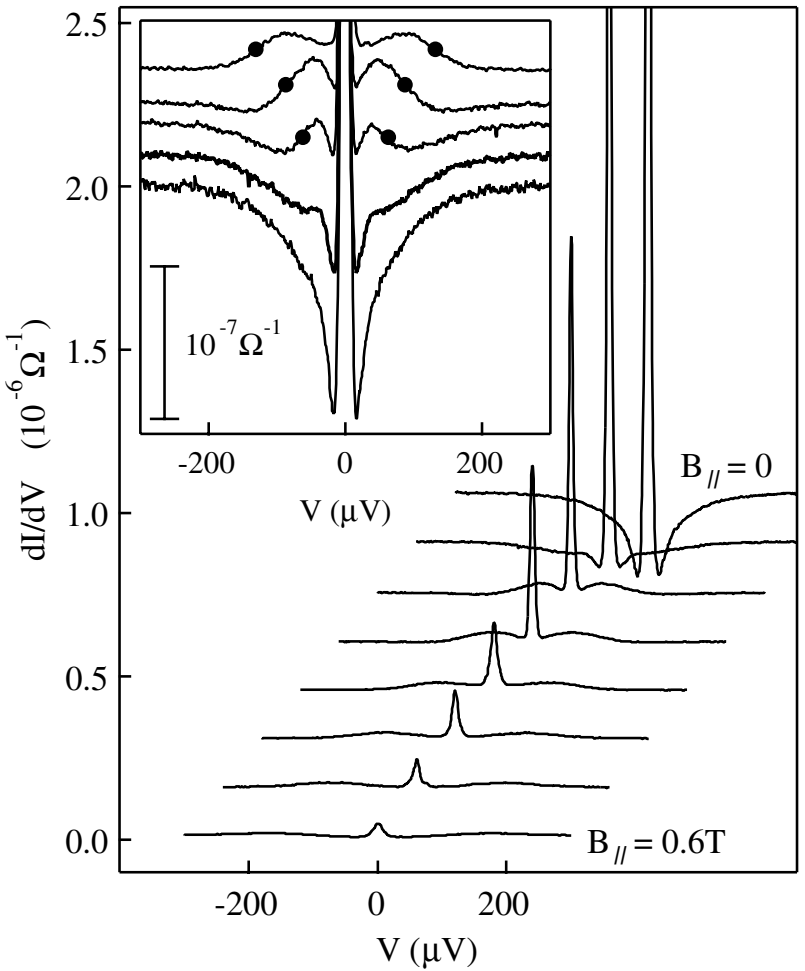

FIG. 3. Tunneling conductance spectra at $T=25 \mathrm{mK}$ and $N_{T}=5.2 \times 10^{10} \mathrm{~cm}^{-2}$ for various parallel magnetic fields. Main panel: $B_{\|}=0,0.11,0.24,0.29,0.35,0.43,0.49$, and $0.59 \mathrm{~T}$. Inset: expanded view of spectra for $B_{\|}=0.07,0.11$, $0.15,0.24$, and $0.35 \mathrm{~T}$. Dots indicate the positions of the split-off resonances in $d I / d V$.

"derivative" shape theoretically expected $[15,16]$. With this in mind, we identify the energy of the resonances with the voltage $V^{*}$ at which the derivative of the conductance, i.e., $d^{2} I / d V^{2}$, exhibits an extremum. The solid dots in the inset in Fig. 3 show this identification. At high $B_{\|}$these intriguing resonances are lost in the broad, presumably incoherent, tunneling background.

Figure 4 displays the average energy $e V^{*}$ of the split-off resonances versus the wave vector $q=e B_{\|} d / \hbar$. Data for three different densities, $N_{T}=5.2,6.0$, and $6.4 \times$ $10^{10} \mathrm{~cm}^{-2}$, corresponding to $d / \ell=1.61,1.71$, and 1.76 , are shown. Within the uncertainties these data lie on straight lines whose slopes imply a velocity $c$ of about $1.4 \times 10^{4} \mathrm{~m} / \mathrm{s}$. We believe that these experimental results demonstrate the existence of a linearly dispersing collective mode in the bilayer $2 \mathrm{D}$ electron system at $\nu_{T}=1$. This mode is very likely the anticipated pseudospin Goldstone mode of the broken symmetry state. The dashed line in Fig. 4 shows a recent theoretical estimate of the dispersion relation of this mode at long wavelengths [20].

The data in Fig. 3 also possess aspects which are not explained by the theoretical models $[15,16]$ mentioned above. The biggest puzzle is presented by the residual zero bias conductance peak which persists to significant $B_{\|}$. This feature is not contained within present perturbative theories 


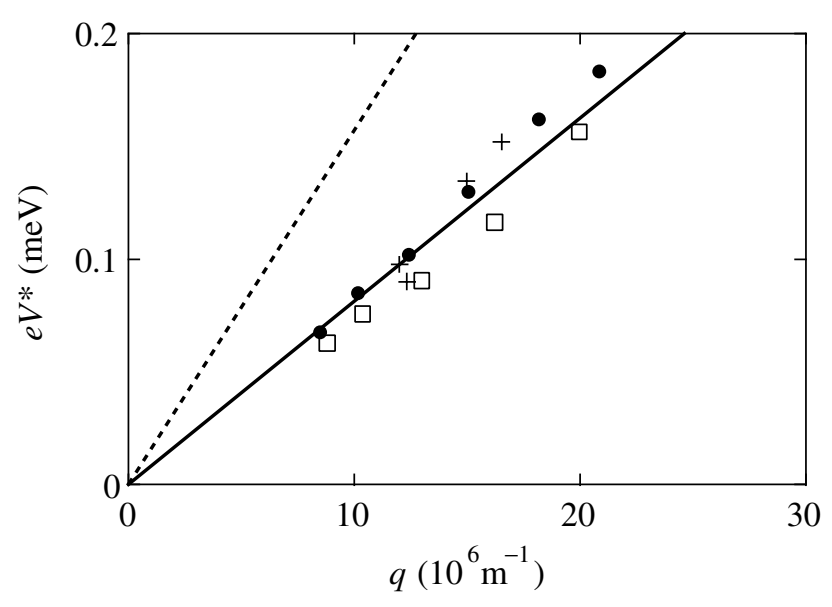

FIG. 4. Energy, $e V^{*}$, of split-off peaks vs the wave vector $q=$ $e B_{\|} d / \hbar$ induced by the parallel magnetic field. Crosses: $N_{T}=$ $6.4 \times 10^{10} \mathrm{~cm}^{-2}$; empty squares: $N_{T}=6.0 \times 10^{10} \mathrm{~cm}^{-2}$; filled circles: $N_{T}=5.2 \times 10^{10} \mathrm{~cm}^{-2}$. The dashed line is a theoretical estimate [20] for the Goldstone mode dispersion relation at small $q$. The solid line is a guide to the eye and corresponds to a collective mode velocity of $1.4 \times 10^{4} \mathrm{~m} / \mathrm{s}$.

$[15,16]$. One possibility is that this peak reflects second order (in the tunneling amplitude) "two-magnon" processes [21]. Alternatively, the residual zero bias peak might be a nonperturbative effect and one may speculate on its possible relation to a Josephson supercurrent.

An interesting analogy has been drawn [22] between tunneling in this bilayer 2D system and a conventional Josephson junction (JJ). In a classic experiment, Eck, Scalapino, and Taylor [23] observed resonances at finite voltage in the dc tunnel current of a $\mathrm{JJ}$ in the presence of a parallel field $B_{\|}$. These resonances were successfully interpreted as resulting from excitation of the electromagnetic modes (i.e., the Swihart modes) of the junction by the ac Josephson current. At nonzero $B_{\|}$the temporal and spatial oscillations of the Josephson current can resonantly excite the junction modes. This leads to features in the dc tunneling characteristics which trace out the linear dispersion of those modes as the parallel field is varied. In the present case the analogous mode is the pseudospin Goldstone mode and the corresponding excitation is produced by the periodic tunneling currents which result from the ferromagnetic order within the bilayer 2DES at finite $B_{\|}[21]$.

In summary, magnetotunneling spectroscopy experiments on double layer 2D electron systems in the $\nu_{T}=1$ QHE state reveal a collective mode in the system which disperses linearly with wave vector at low energy. The measured velocity of this mode is in reasonable agreement with theoretical estimates for the Goldstone mode of the broken symmetry ground state. The question of whether the system supports a Josephson effect remains open.

It is a pleasure to acknowledge fruitful discussions with Steve Girvin, Allan MacDonald, Ady Stern, and Misha Fogler. This work was supported by the NSF under Grant No. DMR0070890 and the DOE under Grant No. DEFG03-99ER45766. One of us (I. B. S.) acknowledges support from the Department of Defense.

[1] For an early example, see L. V. Keldysh and Y. V. Kopaev, Fiz. Tverd. Tela (Leningrad) 6, 2791 (1964) [Sov. Phys. Solid State 6, 2219 (1965)].

[2] For reviews of the quantum Hall effect in double layer systems, see S. M. Girvin and A. H. MacDonald, in Perspectives in Quantum Hall Effects, edited by S. Das Sarma and A. Pinczuk (John Wiley, New York, 1997), Chap. 5; J. P. Eisenstein, ibid., Chap. 2.

[3] Kun Yang et al., Phys. Rev. Lett. 72, 732 (1994); K. Moon et al., Phys. Rev. B 51, 5138 (1995).

[4] A. Stern, S. Das Sarma, M. P. A. Fisher, and S. M. Girvin, Phys. Rev. Lett. 84, 139 (2000).

[5] X. G. Wen and A. Zee, Phys. Rev. Lett. 69, 1811 (1992); Phys. Rev. B 47, 2265 (1993).

[6] Z. F. Ezawa and A. Iwazaki, Phys. Rev. B 47, 7295 (1993).

[7] I. B. Spielman, J. P. Eisenstein, L. N. Pfeiffer, and K. W. West, Phys. Rev. Lett. 84, 5808 (2000).

[8] The true spins are assumed to be fully polarized by the Zeeman energy.

[9] J. Schliemann, S. M. Girvin, and A. H. MacDonald, Phys. Rev. Lett. 86, 1849 (2001).

[10] E. Demler, C. Nayak, and S. Das Sarma, Phys. Rev. Lett. 86, 1853 (2001).

[11] Y. B. Kim, C. Nayak, E. Demler, N. Read, and S. Das Sarma, cond-mat/0011459.

[12] H. Fertig, Phys. Rev. B 40, 1087 (1989).

[13] J. P. Eisenstein, L. N. Pfeiffer, and K. W. West, Phys. Rev. Lett. 69, 3804 (1992).

[14] S. Q. Murphy, J. P. Eisenstein, L. N. Pfeiffer, and K. W. West, Phys. Rev. B 52, 14825 (1995).

[15] A. Stern, S. M. Girvin, A. H. MacDonald, and N. Ma, Phys. Rev. Lett. 86, 1829 (2001).

[16] L. Balents and L. Radzihovsky, Phys. Rev. Lett. 86, 1825 (2001).

[17] See, for example, D. J. Scalapino, Phys. Rev. Lett. 24, 1052 (1970).

[18] J. Smoliner et al., Phys. Rev. Lett. 63, 2116 (1989).

[19] J.P. Eisenstein, T. J. Gramila, L. N. Pfeiffer, and K. W. West, Phys. Rev. B 44, 6511 (1991).

[20] A. H. MacDonald (private communication); Ref. [3].

[21] S. M. Girvin (private communication).

[22] M. Fogler and F. Wilczek, Phys. Rev. Lett. 86, 1833 (2001).

[23] R. E. Eck, D. J. Scalapino, and B. N. Taylor, Phys. Rev. Lett. 13, 15 (1964). 